\title{
Chaotic planar piecewise smooth vector fields with non-trivial minimal sets
}

\author{
CLAUDIO A. BUZZI $\dagger$, TIAGO DE CARVALHO $\ddagger$ and RODRIGO D. EUZÉBIO $\dagger$ \\ $\dagger$ IBILCE-UNESP, CEP 15054-000, S. J. Rio Preto, São Paulo, Brazil \\ (e-mail: buzzi@ibilce.unesp.br, rodrigo.euzebio@sjrp.unesp.br) \\ $\ddagger$ FC-UNESP, CEP 17033-360, Bauru, São Paulo, Brazil \\ (e-mail: tcarvalho@fc.unesp.br)
}

(Received 1 February 2014 and accepted in revised form 11 June 2014)

\begin{abstract}
In this paper some aspects on chaotic behavior and minimality in planar piecewise smooth vector fields theory are treated. The occurrence of non-deterministic chaos is observed and the concept of orientable minimality is introduced. Some relations between minimality and orientable minimality are also investigated and the existence of new kinds of non-trivial minimal sets in chaotic systems is observed. The approach is geometrical and involves the ordinary techniques of non-smooth systems.
\end{abstract}

\section{Introduction}

Piecewise smooth vector fields (PSVFs) have become certainly one of the common frontiers between mathematics and physics or engineering. Many authors have contributed to the study of PSVFs (see for instance the pioneering work [5] or the didactic works $[\mathbf{1}, \mathbf{1 0}]$, and references therein about details of these multi-valued vector fields). In our approach Filippov's convention is considered. So, the vector field of the model is discontinuous across a switching manifold and it is possible for its trajectories to be confined onto the switching manifold itself. The occurrence of such behavior, known as sliding motion, has been reported in a wide range of applications. We can find important examples in electrical circuits having switches, in mechanical devices in which components collide with each other, in problems with friction, sliding or squealing, among others (see [1]).

For smooth vector fields there is a very developed theory nowadays, mainly in the planar case. In such environment, questions about chaotic behavior and minimality, for instance, are completely answered. Indeed, the Jordan curve theorem assures that there is no chaotic behavior in planar systems and the Poincaré-Bendixson theorem says that for a given flow the minimal sets are just equilibria or limit cycles. Nevertheless, in higher dimension, while minimal sets are described by the Denjoy-Schwartz theorem (under some suitable 
hypothesis; see [7]), chaotic systems have been intensively studied and yet a final theory is still far from being reached.

A very interesting and useful subject is to study these kind of objects in the PSVF scenario. Furthermore, we must observe that chaotic behavior and non-trivial minimality have been little studied in the PSVF literature. In three-dimensional systems, some results on chaotic behavior were obtained by Colombo and Jeffrey $[\mathbf{3}, \mathbf{8}]$. As far as we know, chaos has not been treated in planar PSVFs apart from the particular example exhibited in [8]. In addition, some questions on non-trivial minimality were provided in [2] for planar PSVFs.

The specific topic addressed in this paper concerns the occurrence of chaos in planar PSVFs and some distinct definitions of minimal sets. Moreover, we study the occurrence of non-trivial minimal sets different from those presented in [2]. We stress that a trivial minimal set is either an equilibrium point or a closed periodic orbit. For smooth vector fields this is a very important subject because minimal sets are an essential part of limit sets. As far as the authors know, a first study about the minimal set theory for PSVFs and a discussion about the validity of the Poincaré-Bendixson theorem for PSVFs is given only in our previous paper [2]. In [2] we gave a definition of minimal sets for the PSVF scenario and exhibited non-trivial minimal sets for such systems with sliding motion. Moreover, we showed that an analogous theorem to the Poincaré-Bendixson theorem for PSVFs without sliding motion can be achieved.

Following the approach in [2], here we present some special PSVFs and prove the existence of compact invariant sets with chaotic flow. Actually, these sets will be nontrivial minimal sets having no symmetry. We also propose definitions of minimal sets for positive (and negative) flow of PSVFs (or orientable minimality) and study some relations between them and the definition of a minimal set established in [2]. With these new definitions we analyze the occurrence of new kind of non-trivial minimal sets for PSVFs defined in $\mathbb{R}^{2}$ and the validity of a theorem analogous to the Poincaré-Bendixson theorem.

The paper is organized as follows: in $\S 2$ some concepts of the standard theory on PSVFs and a brief introduction about Filippov systems are introduced. In $\S 3$ the results of the paper are presented into two parts: first, in $\$ 3.1$, the occurrence of chaos in a particular set is verified. Later, in $\$ 3.2$ definitions of minimal sets for positive (and negative) flow of PSVFs are established, some correspondences between them are studied and the presence of chaotic behavior in some minimal sets under these definitions are examined. Moreover, a theorem relating orientable minimality and chaos is presented. Finally, in $\S 3.3$ a short conclusion of the work developed in the present paper is presented.

\section{Preliminaries}

Let $V$ be an arbitrarily small neighborhood of $0 \in \mathbb{R}^{2}$ and consider a codimension-one manifold $\Sigma$ of $\mathbb{R}^{2}$ given by $\Sigma=f^{-1}(0)$, where $f: V \rightarrow \mathbb{R}$ is a smooth function having $0 \in \mathbb{R}$ as a regular value (i.e. $\nabla f(p) \neq 0$, for any $p \in f^{-1}(0)$ ). We call $\Sigma$ the switching manifold that is the separating boundary of the regions $\Sigma^{+}=\{q \in V \mid f(q) \geq 0\}$ and $\Sigma^{-}=\{q \in V \mid f(q) \leq 0\}$. Observe that we can assume, locally around the origin of $\mathbb{R}^{2}$, that $f(x, y)=y$. 


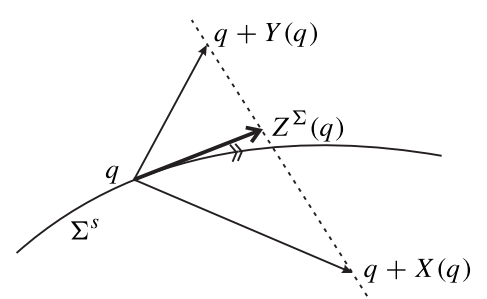

FIGURE 1. Filippov's convention.

Designate by $\chi$ the space of $C^{r}$-vector fields on $V \subset \mathbb{R}^{2}$, with $r \geq 1$ large enough for our purposes. Call $\Omega$ the space of vector fields $Z: V \rightarrow \mathbb{R}^{2}$ such that

$$
Z(x, y)= \begin{cases}X(x, y) & \text { for }(x, y) \in \Sigma^{+}, \\ Y(x, y) & \text { for }(x, y) \in \Sigma^{-},\end{cases}
$$

where $X=\left(X_{1}, X_{2}\right), Y=\left(Y_{1}, Y_{2}\right) \in \chi$. The trajectories of $Z$ are solutions of $\dot{q}=Z(q)$ and we accept it to be multi-valued at points of $\Sigma$. The basic results of differential equations in this context were stated by Filippov in [5], that we summarize next. Indeed, consider Lie derivatives

$$
X \cdot f(p)=\langle\nabla f(p), X(p)\rangle \quad \text { and } \quad X^{i} \cdot f(p)=\left\langle\nabla X^{i-1} \cdot f(p), X(p)\right\rangle, \quad i \geq 2
$$

where $\langle.,$.$\rangle is the usual inner product in \mathbb{R}^{2}$.

We distinguish the following regions on the discontinuity set $\Sigma$ :

(i) $\Sigma^{c} \subseteq \Sigma$ is the sewing region if $(X \cdot f)(Y \cdot f)>0$ on $\Sigma^{c}$;

(ii) $\Sigma^{e} \subseteq \Sigma$ is the escaping region if $(X \cdot f)>0$ and $(Y \cdot f)<0$ on $\Sigma^{e}$;

(iii) $\quad \Sigma^{s} \subseteq \Sigma$ is the sliding region if $(X \cdot f)<0$ and $(Y \cdot f)>0$ on $\Sigma^{s}$.

The sliding vector field associated to $Z \in \Omega$ is the vector field $Z^{s}$ tangent to $\Sigma^{s}$ and defined at $q \in \Sigma^{s}$ by $Z^{s}(q)=m-q$ with $m$ being the point of the segment joining $q+$ $X(q)$ and $q+Y(q)$ such that $m-q$ is tangent to $\Sigma^{s}$ (see Figure 1). It is clear that if $q \in$ $\Sigma^{s}$, then $q \in \Sigma^{e}$ for $-Z$ and then we can define the escaping vector field on $\Sigma^{e}$ associated to $Z$ by $Z^{e}=-(-Z)^{s}$. In what follows we use the notation $Z^{\Sigma}$ for both cases. In our pictures we represent the dynamics of $Z^{\Sigma}$ by double arrows.

In what follows we present the definition of local and global trajectories for PSVFs. Before that, we remark that a tangency point of system (1) is characterized by $(X . f(q))(Y . f(q))=0$. If there exist an orbit of the vector field $X$ (or $Y$ ) reaching $q$ in a finite time, then such tangency is called a visible tangency for $X$ (respectively $Y$ ). Otherwise we call $q$ an invisible tangency for $X$ (respectively $Y$ ). In addition, a tangency point $p$ is singular if $p$ is a invisible tangency for both $X$ and $Y$. On the other hand, a tangential singularity $p$ is regular if it is not singular.

The definition of local trajectory can also be found in [6].

Definition 1. The local trajectory (orbit) $\phi_{Z}(t, p)$ of a PSVF given by (1) is defined as follows.

- For $p \in \Sigma^{+} \backslash \Sigma$ and $p \in \Sigma^{-} \backslash \Sigma$ the trajectory is given by $\phi_{Z}(t, p)=\phi_{X}(t, p)$ and $\phi_{Z}(t, p)=\phi_{Y}(t, p)$ respectively, where $t \in I$. 
- $\quad$ For $p \in \Sigma^{c}$ such that $X \cdot f(p)>0, Y \cdot f(p)>0$ and taking the origin of time at $p$, the trajectory is defined as $\phi_{Z}(t, p)=\phi_{Y}(t, p)$ for $t \in I \cap\{t \leq 0\}$ and $\phi_{Z}(t, p)=$ $\phi_{X}(t, p)$ for $t \in I \cap\{t \geq 0\}$. For the case $X \cdot f(p)<0$ and $Y \cdot f(p)<0$ the definition is the same reversing time.

- For $p \in \Sigma^{e}$ and taking the origin of time at $p$, the trajectory is defined as $\phi_{Z}(t, p)=\phi_{Z^{\Sigma}}(t, p)$ for $t \in I \cap\{t \leq 0\}$ and $\phi_{Z}(t, p)$ is either $\phi_{X}(t, p)$ or $\phi_{Y}(t, p)$ or $\phi_{Z^{\Sigma}}(t, p)$ for $t \in I \cap\{t \geq 0\}$. For the case $p \in \Sigma^{s}$ the definition is the same reversing time.

- $\quad$ For $p$ a regular tangency point and taking the origin of time at $p$, the trajectory is defined as $\phi_{Z}(t, p)=\phi_{1}(t, p)$ for $t \in I \cap\{t \leq 0\}$ and $\phi_{Z}(t, p)=\phi_{2}(t, p)$ for $t \in$ $I \cap\{t \geq 0\}$, where each $\phi_{1}, \phi_{2}$ is either $\phi_{X}$ or $\phi_{Y}$ or $\phi_{Z^{\Sigma}}$.

- $\quad$ For $p$ a singular tangency point $\phi_{Z}(t, p)=p$ for all $t \in \mathbb{R}$.

The following definitions were stated in [2].

Definition 2. A global trajectory (orbit) $\Gamma_{Z}\left(t, p_{0}\right)$ of $Z \in \chi$ passing through $p_{0}$ is a union

$$
\Gamma_{Z}\left(t, p_{0}\right)=\bigcup_{i \in \mathbb{Z}}\left\{\sigma_{i}\left(t, p_{i}\right) \mid t_{i} \leq t \leq t_{i+1}\right\}
$$

of preserving-orientation local trajectories $\sigma_{i}\left(t, p_{i}\right)$ satisfying $\sigma_{i}\left(t_{i+1}, p_{i}\right)=\sigma_{i+1}$ $\left(t_{i+1}, p_{i+1}\right)=p_{i+1}$ and $t_{i} \rightarrow \pm \infty$ as $i \rightarrow \pm \infty$. A global trajectory is a positive (respectively, negative) global trajectory if $i \in \mathbb{N}$ (respectively, $-i \in \mathbb{N}$ ) and $t_{0}=0$.

Definition 3. A set $A \subset \mathbb{R}^{2}$ is invariant for $Z$ if for each $p \in A$ and all global trajectory $\Gamma_{Z}(t, p)$ passing through $p$ it holds $\Gamma_{Z}(t, p) \subset A$.

Definition 4. Consider $\mathbf{Z} \in \Omega$. A set $M \subset \mathbb{R}^{2}$ is minimal for $Z$ if:

(i) $\quad M \neq \emptyset$;

(ii) $M$ is compact;

(iii) $M$ is invariant for $Z$;

(iv) $M$ does not contain proper subset satisfying (i)-(iii).

In the next section we present the main results of the paper.

\section{Main results}

3.1. Non-deterministic chaos in planar PSVFs. Since the dynamic on sliding and escaping regions are set-valued, following the previous nomenclature of [3] and [9], it is non-deterministic. In fact, as far as we know, the definition of non-deterministic chaos for PSVFs was first introduced in [3], where the authors adapt the classical definition of, for example [9], to this context. Of course, the definition must contemplate topological transitivity and sensitivity to initial conditions. For this purpose, consider the following definitions.

Definition 5. System (1) is topologically transitive on an invariant set $W$ if for every pair of non-empty, open sets $U$ and $V$ in $W$, there exist $q \in U, \Gamma_{Z}^{+}(t, q)$ a positive global trajectory and $t_{0}>0$ such that $\Gamma_{Z}^{+}\left(t_{0}, q\right) \in V$. 


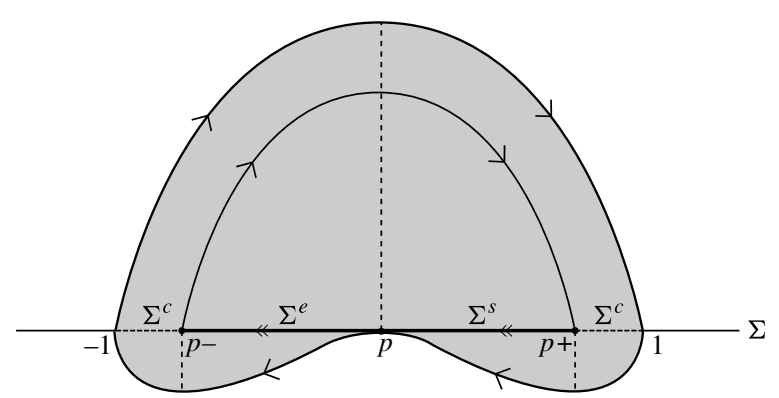

FIgURE 2. The minimal set $\Lambda$. The PSVF $Z=\left((1,-2 x),\left(-2,4 x^{3}-2 x\right)\right)$ is chaotic on it.

Definition 6. System (1) exhibits sensitive dependence on a compact invariant set $W$ if there is a fixed $r>0$ satisfying $r<\operatorname{diam}(W)$ such that for each $x \in W$ and $\varepsilon>0$ there exist a $y \in B_{\varepsilon}(x) \cap W$ and positive global trajectories $\Gamma_{x}^{+}$and $\Gamma_{y}^{+}$passing through $x$ and $y$, respectively, satisfying

$$
d_{H}\left(\Gamma_{x}^{+}, \Gamma_{y}^{+}\right)=\sup _{a \in \Gamma_{x}^{+}, b \in \Gamma_{y}^{+}} d(a, b)>r
$$

where $\operatorname{diam}(W)$ is the diameter of $W$ and $d$ is the Euclidean distance.

As observed in [3], the two previous definitions coincide with those used for smooth systems when the flow is single-valued, making this a natural extension for a set-valued flow. Now we define a non-deterministic chaotic set.

Definition 7. System (1) is chaotic on a compact invariant set $W$ if it is topologically transitive and exhibits sensitive dependence on $W$.

In what follows we present a chaotic set coming from a non-trivial minimal set.

Theorem 8. Consider $Z=(X, Y) \in \Omega, \quad$ where $X(x, y)=(1,-2 x), \quad Y(x, y)=$ $\left(-2,4 x^{3}-2 x\right)$ and $\Sigma=f^{-1}(0)=\left\{(x, y) \in \mathbb{R}^{2} \mid y=0\right\}$. Then the planar PSVF $Z$ is chaotic (see Figure 2) on the compact invariant set

$$
\Lambda=\left\{(x, y) \in \mathbb{R}^{2} \mid-1 \leq x \leq 1 \text { and } x^{4} / 2-x^{2} / 2 \leq y \leq 1-x^{2}\right\}
$$

Before proving Theorem 8 we present the following lemma. It will be fundamental in the proof of Theorem 8 .

Lemma 1. Consider the set $\Lambda$ defined in Theorem 8. Then, for any $x, y \in \Lambda$, there exist a positive global trajectory $\Gamma^{+}(t, x)$ passing through $x$ and $t_{0}>0$ such that $\Gamma^{+}\left(t_{0}, x\right)=y$.

The previous lemma says that any two points in $\Lambda$ can be connected by some positive global trajectory. Its proof is straightforward if we observe that a global trajectory of any point in $\Lambda$ meets $p$ for some time $t^{*}$, as the authors argued in [2]. Now we prove Theorem 8.

Proof of Theorem 8. In order to prove that the PSVF $Z$ is topologically transitive on $\Lambda$, we observe that $\Lambda$ is compact and invariant since it is minimal (see [2, Proposition 1]). Now 
consider non-empty open sets $U$ and $V$ in $\Lambda$. Since $U$ and $V$ are non-empty, there exist at least an element $\lambda_{1}$ in $U$ and another one $\lambda_{2}$ in $V$. By Lemma 1, there exist a positive global trajectory $\Gamma^{+}\left(t, \lambda_{1}\right)$ passing through $\lambda_{1}$ and $t_{0}>0$ such that $\Gamma^{+}\left(t_{0}, \lambda_{1}\right)=\lambda_{2} \in V$. Consequently the PSVF $Z$ is topologically transitive on the invariant set $\Lambda$.

Now we prove that $Z$ exhibits sensitive dependence on $\Lambda$. Indeed, take $m=\operatorname{diam}(\Lambda)$ and consider $r=m / 2>0$. Since $r<m$, then there exists two elements $a$ and $b$ in $\Lambda$ such that $d(a, b)>r$. Now consider $x \in \Lambda, \varepsilon>0$ and fix $y \in B_{\varepsilon}(x) \cap \Lambda$. By Lemma 1 there exist positive global trajectories $\Gamma_{Z}^{+}(t, x)$ of $x$ and $\Gamma_{Z}^{+}(t, y)$ of $y$ and numbers $t_{1}, t_{2}>0$ such that $\Gamma_{Z}^{+}\left(t_{1}, x\right)=a$ and $\Gamma_{Z}^{+}\left(t_{2}, y\right)=b$. Then $d_{H}\left(\Gamma_{Z}^{+}\left(t_{1}, x\right), \Gamma_{Z}^{+}\left(t_{2}, y\right)\right)=d(a, b)>r$ and consequently $Z$ exhibits sensitive dependence on $\Lambda$. Thus, the planar PSVF $Z$ is chaotic on the invariant compact set $\Lambda$.

We should note that apart of topologically transitive and sensitive dependence, the classical definition of chaos given by Devaney in [4] demands also that periodic trajectories of the considered system are dense in $\Lambda$. However, system $Z$ exhibited in Theorem 8 also presents such a property, as we can see in Theorem 10. Before that, we introduce the notion of periodic trajectory for PSVF. Actually, it is analogous to the definition of periodic trajectory for smooth systems.

Definition 9. Let $\Gamma_{Z}(t, q)$ a global trajectory of the PSVF $Z=(X, Y)$. We say that $\Gamma_{Z}$ is periodic if $\Gamma_{Z}$ is periodic in the variable $t$, i.e. if there exist $T>0$ such that $\Gamma_{Z}(t+T, q)=$ $\Gamma_{Z}(t, p)$, for all $t \in \mathbb{R}$.

THEOREM 10. Consider $Z$ and $\Lambda$ as presented in Theorem 8. The periodic trajectories of $Z$ are dense in $\Lambda$.

Proof. The proof is completed if we show that for every point $x \in \Lambda$ passes a periodic trajectory of $Z$. In order to see that, consider $\sigma_{0}$ the closed arc connecting point $x$ with itself $\left(\sigma_{0} \neq\{x\}\right)$. The existence of such arc is due to Lemma 1 . Then the global trajectory

$$
\Gamma_{Z}(t, x)=\bigcup_{i \in \mathbb{Z}}\left\{\sigma_{i}(t, x) \mid t_{i} \leq t \leq t_{i+1}\right\}
$$

satisfying $\sigma_{i}=\sigma_{0}$ for all $i \in \mathbb{Z}$ is $t_{1}$-periodic and passes through $x$. Observe that $\sigma_{i}\left(k t_{1}, x\right)=x$, for all $k \in \mathbb{Z}$ and for all $i \in \mathbb{Z}$.

The concepts of chaos in PSVF are also discussed through the next section, where we present new examples of chaotic PSVF and an interesting relation between chaotic PSVF and orientable minimality.

3.2. Orientable minimality and chaos. In the recent theory of PSVFs there exist a lot of examples where the dynamics in the non-smooth context is richer than their smooth analogous. It happens basically due to the non-existence of a theorem that assure the uniqueness of a trajectory crossing the switching manifold. In fact, the orbit passing through a sliding or escaping segment on the switching manifold can run out from such segment when the time goes to future or past. Supported by these facts, in the present section we introduce some definitions concerning minimal sets by distinguishing 
invariance for positive and negative global trajectories. That is what we call orientable minimality. The advantage by taking into account such approach is to consider some interesting sets that are not properly minimal but also present invariance and compactness in some sense.

In addition, we will verify the existence of some minimal sets that do not have a canard structure, i.e. coincidence of a visible and an invisible tangencies separating a sliding from an escaping region on the switching manifold. In particular, it means that the conditions in order to find a non-trivial minimal set are not so strong as the situation when the canard phenomena happen. Finally, we must observe that some of these new objects also present a chaotic behavior. This emphasizes that, in PSVFs, systems having non-trivial minimal sets and chaotic behavior on them have some intersection, as we can see in Theorem 14.

In what follows we present new definitions on invariance and minimality. Then we compare some special sets taking into account such definitions.

Definition 11. A set $A \subset \mathbb{R}^{2}$ is positive-invariant (respectively, negative-invariant) if for each $p \in A$ and all positive global trajectory $\Gamma_{Z}^{+}(t, p)$ (respectively, negative global trajectory $\Gamma_{Z}^{-}(t, p)$ ) passing through $p$ it holds $\Gamma_{Z}^{+}(t, p) \subset A$ (respectively, $\left.\Gamma_{Z}^{-}(t, p) \subset A\right)$.

Remark 1. It follows directly from Definition 11 that a given set is invariant if and only if it is positive-invariant and negative-invariant.

Definition 12. Consider $\mathbf{Z} \in \Omega$. A set $M \subset \mathbb{R}^{2}$ is positive-minimal (respectively, negativeminimal) if:

(i) $\quad M \neq \emptyset$;

(ii) $M$ is compact;

(iii) $M$ is positive-invariant (respectively, negative-invariant);

(iv) $M$ does not contain proper subset satisfying (i)-(iii).

The following lemma is a trivial consequence of Definition 12.

LEMma 2. Consider $M \in \mathbb{R}^{2}$ and $Z$ a PSVF. If $M$ is positive-minimal and negativeminimal, then $M$ is minimal.

Proof. In fact, since $M$ is positive-minimal and negative-minimal, then $M$ is a non-empty compact set and from Remark $1 M$ is invariant and does not contain a proper non-empty compact invariant subset.

Throughout this paper we will see that the converse of Lemma 2 does not holds.

We stress that a canard cycle of kind III is a closed trajectory of $Z$ with, at least, one tangency point of $X$ or $Y$ with the switching manifold (for a precise definition, see [2, Definition 5]). A pseudo-equilibria is any point $q$ such that $Z^{\Sigma}(q)=0$, where $Z^{\Sigma}$ represents the sliding vector field associated to $Z$.

Example 1. We know that canard cycle of kind III and pseudo-equilibria are positive or negative invariants compact sets, but they are not positive and negative at the same time. It holds due to Definition 1 of local trajectories. Consequently they are positive-minimal or negative-minimal, depending on the orientation of the trajectories, but not minimal. 


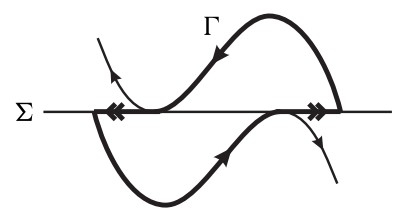

FIGURE 3. Canard cycle of kind III.

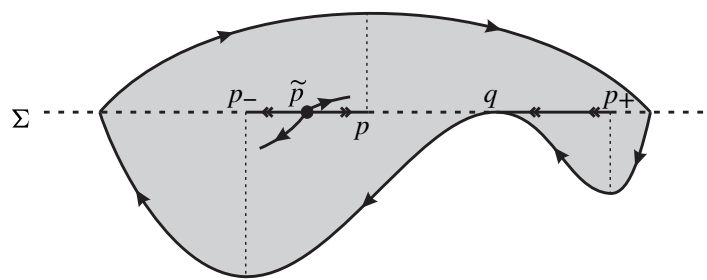

FIgURE 4. The minimal set $\Lambda_{1}$ for $Z_{1}$. Here $\Lambda_{1}$ is neither positive-minimal nor negative-minimal.

In [2] is exhibited examples of minimal sets of PSVFs. In those examples it is always verified the occurrence of fold-fold singularities (which is characterized by the coincidence of a fold point of $X$ and a fold point of $Y$ ); however, at the next example of a minimal set this fact is not required.

Example 2. Consider $Z_{1}=(X, Y) \in \Omega$, where $X(x, y)=(1,-2 x+1), \quad Y(x, y)=$ $(-1,(-2+x)(-22+x(-7+4 x)))$ and $\Sigma=f^{-1}(0)=\left\{(x, y) \in \mathbb{R}^{2} \mid y=0\right\}$. The parametric equation for the integral curves of $X$ and $Y$ with initial conditions $(x(0), y(0))=\left(0, k_{+}\right)$and $(x(0), y(0))=\left(0, k_{-}\right)$, respectively, are known and their algebraic expressions are given by $y=-(-4+x)(3+x)+k_{+}$and $y=$ $(-4+x)(-2+x)^{2}(3+x)+k_{-}$, respectively. It is easy to see that $p=(1 / 2,0)$ is an invisible tangency point of $X, q=(2,0)$ is a visible tangency point of $Y$ and the points $p_{ \pm}=((7 \pm \sqrt{401}) / 8,0)$ are both invisible tangency points of $Y$. Note that, in $\Sigma$, between $p_{-}$and $p$ there exists an escaping region with a repeller pseudo equilibrium $\tilde{p}$ on it and between $q$ and $p_{+}$there exists a sliding region. Further, every point between $(-3,0)$ and $p_{-}$or between $p$ and $q$ belong to a sewing region. Consider now the particular trajectories of $X$ and $Y$ for the cases when $k_{+}=0$ and $k_{-}=0$, respectively. These particular curves delimit a bounded region of plane that we call $\Lambda_{1}$. Figure 4 summarizes these facts.

Example 2 leads to the next proposition.

Proposition 1. Consider $Z_{1}=(X, Y) \in \Omega$, where $X(x, y)=(1,-2 x+1), Y(x, y)=$ $(-1,(-2+x)(-22+x(-7+4 x)))$ and $\Sigma=f^{-1}(0)=\left\{(x, y) \in \mathbb{R}^{2} \mid y=0\right\}$. The set

$$
\begin{aligned}
\Lambda_{1} & =\left\{(x, y) \in \mathbb{R}^{2} \mid-3 \leq x \leq 4 \text { and }(-4+x)(-2+x)^{2}(3+x) \leq y\right. \\
& \leq-(-4+x)(3+x)\} .
\end{aligned}
$$

is minimal for $Z_{1}$ but it is neither positive-minimal nor negative-minimal.

Proof. It is easy to see that $\Lambda_{1}$ is compact and has non-empty interior. In order to see that $\Lambda_{1}$ is invariant it is enough to observe that, by Definition 1 , on $\partial \Lambda_{1} \backslash\{q\}$ we have 


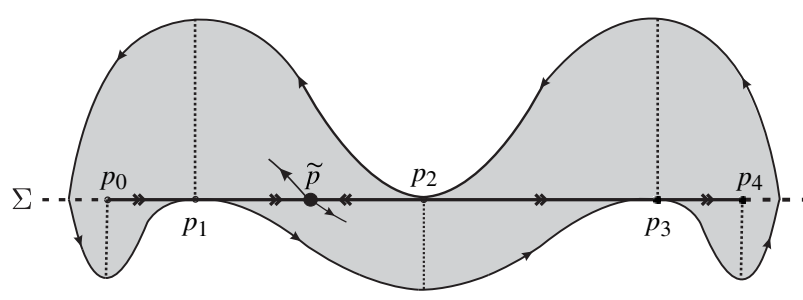

FIGURE 5. The minimal set $\Lambda_{2}$ for $Z_{2}$.

uniqueness of trajectory (here $\partial B$ means the boundary of the set $B$ ). Since $q$ is a visible tangency point for $Y$, according to the fourth bullet of Definition 1 any trajectory passing through $q$ remain in $\Lambda_{1}$. Consequently $\Lambda_{1}$ is invariant.

The trajectory of $X$ passing through $p_{-}$meets $\Sigma$ at $p_{-}^{1}=((1+\sqrt{401}) / 8,0)$, we conclude that the global trajectory of any point in $\Lambda_{1}$ meets $q$ for some time $t^{*}$. Let $\Lambda_{1}^{\prime} \subset \Lambda_{1}$ be a invariant set. Then, by the invariance of $\Lambda_{1}^{\prime}$ and the previous comment it is clear that $q \in \Lambda_{1}^{\prime}$. Now take a point $u \in \Lambda_{1}$ and note that there exists a time $t_{u}>0$ for which the positive trajectory $\Gamma^{+}(t, u)$ through $u$ satisfies $\Gamma^{+}\left(t_{u}, u\right)=q \in \Lambda_{1}^{\prime}$. Then, by the invariance of $\Lambda_{1}^{\prime}$, we have that $u \in \Lambda_{1}^{\prime}$. Therefore, $\Lambda_{1}^{\prime}=\Lambda_{1}$ and then $\Lambda_{1}$ is minimal for $Z_{1}$.

Moreover, since $\partial \Lambda_{1}$ is positive-invariant, the set $\Lambda_{1}$ is not positive-minimal and since $\tilde{p}$ is negative-invariant the set $\Lambda_{1}$ is not negative-minimal.

Example 3. The minimal set presented in Theorem 8 is also positive-minimal and negative-minimal. The proof of this fact follows the same lines of the proof of Proposition 1.

The next example is a small variation of the Example 3.

Example 4. Consider $Z_{2}$ a PSVF presenting the phase portrait exhibited in Figure 5. Here, there exists a compact set $\Lambda_{2}$ bounded by trajectories of $X$ and $Y$. As illustrated, $p_{1}$ and $p_{3}$ are invisible tangency points of $X, p_{2}$ is a visible tangency point of $X, p_{1}$ and $p_{3}$ are visible tangency points of $Y$ and $p_{0}, p_{2}$ and $p_{4}$ are invisible tangency points of $Y$. It is easy to see that $\Lambda_{2}$ is invariant and there is not a proper subset which is compact and invariant. So, $\Lambda_{2}$ is minimal for $Z_{2}$. Assume that there exists a pseudo-equilibrium $\tilde{p}$ between $p_{1}$ and $p_{2}$. Following the orientation of the trajectories at Figure 5 and the third bullet of Definition 1 we conclude that $\Lambda_{2}$ is not negative-minimal since $\{\tilde{p}\}$ is a compact negative-invariant set. Moreover, $\Lambda_{2}$ is positive-minimal since it is positive-invariant and it have not a compact proper subset which is positive-invariant.

Proposition 2. Consider the notation of Example 4. The set $\Lambda_{2}$ is minimal for $Z_{2}$ and also positive-minimal but not negative-minimal for this PSVF.

Proof. Straightforward, following the argumentation on Example 4.

The next remark is an analogous of Proposition 2 by considering the opposite orientation of the time. 
Remark 2. Consider a PSVF presenting the phase portrait exhibited in Figure 5 with opposite orientation and the notation of Example 4. Then, following the same ideas of this example, we obtain that $\Lambda_{2}$ is minimal for $Z_{2}$ and negative-minimal but not positiveminimal for this PSVF.

The previous propositions, examples and remarks of this subsection say that non-trivial minimality can occur in non-symmetrical sets having no canard points. We must note that the example exhibited in [8] of a chaotic planar system on a special set neither present symmetry nor the set is minimal. Moreover, in our examples we can observe some similarity between symmetrical minimal sets and systems that are positive-minimal and negative-minimal simultaneously. Also, by observing Examples 1-4 we note that the presence of sliding and escaping regions on $\Sigma$ generates many different objects with very rich dynamics. We should note that the case where do not exist sliding or escape regions was studied in [2] and was shown that in such case there are no non-trivial minimal sets. In particular, we see that even considering different definitions of minimal sets and nonsymmetrical sets having no canard points we cannot generalize the classical PoincaréBendixson theorem to the non-smooth context.

The following result indicates the presence of chaos in the systems studied in this section.

THEOREM 13. Consider the PSVF $Z_{2}$ and the set $\Lambda_{2}$ as presented in Proposition 2. Then $Z_{2}$ is chaotic on $\Lambda_{2}$.

Proof. The proof of Theorem 13 follows the same lines as the proof of Theorem 8 by using a similar result to Lemma 1 for the minimal set $\Lambda_{2}$.

One should note that Theorems 8 and 13 present examples of PSVFs that are chaotic on minimal sets. This fact suggests a relation between chaoticity and minimality in PSVF that we make clear in the following theorem.

In what follows we denote by med $(\cdot)$ the Lebesgue measure.

THEOREM 14. Let $Z$ be a planar PSVF and $\Lambda \subset \mathbb{R}^{2}$ a compact invariant set. If $\Lambda$ is positive-minimal and negative-minimal satisfying $\operatorname{med}(\Lambda)>0$, then $Z$ is chaotic on $\Lambda$.

Theorem 14 is a very interesting result because it presents a connection between two important different objects of the recent theory of PSVF, namely, the chaotic planar systems and the non-trivial minimal sets.

In order to prove Theorem 14, we introduce the next two lemmas. The first is a generalization of Lemma 1.

LEMMA 3. Under the same hypotheses of Theorem 14, it holds that for any $x, y \in \Lambda$, there exist a global trajectory $\Gamma(t, y)$ passing through $y$ and $t^{*}>0$ such that $\Gamma^{+}\left(t^{*}, y\right)=x$.

Proof. Since $\operatorname{med}(\Lambda)>0$, by the Poincaré-Bendixson theorem for PSVF presented in [2], there exist at least a set $A \subset \Sigma \cap\left(\Sigma^{e} \cup \Sigma^{s}\right)$. Otherwise, we have $\Sigma \cap \Lambda=\Sigma^{c} \cup \Sigma^{t}$ and then by the referred theorem we get $\operatorname{med}(\Lambda)=0$, where $\Sigma^{t}$ is the set of tangencies 
points of $Z$. For each $a \in A$, denote by $\Pi_{a}^{+}$the set of all positive global trajectories passing through $a$ and by $\Pi_{a}^{-}$its negative analog. Now consider the sets

$$
A_{a}^{ \pm}=\bigcup_{\Gamma_{a} \in \Pi_{a}^{ \pm}} \Gamma_{a}(t, a) \subset \Lambda .
$$

Actually we have $A_{a}^{ \pm}=\Lambda$, since $A_{a}^{ \pm}$is positive-invariant (respectively negative-invariant) restrained in the positive-minimal (respectively negative-minimal) set $\Lambda$. In order to see that $A_{a}^{+}$is positive-invariant, let $p$ be a point in $A_{a}^{+}$and $\Gamma_{p}(t, p)$ a positive global trajectory passing through $p$. Since $p \in A_{a}^{+}$, then there exists a positive global trajectory $\tilde{\Gamma}_{a}(t, a)$ passing through $a$ and $t_{0}>0$ such that $\tilde{\Gamma}_{a}\left(t_{0}, a\right)=p$. Consequently $\Gamma_{p}(t, p)$ belongs to $A_{a}^{+}$once it is restrained to the positive global trajectory $\hat{\Gamma}_{a}(t, a)=\tilde{\Gamma}_{a}(t, a) \cup \Gamma_{p}(t, p) \subset$ $A_{a}^{+}$. Analogously we can prove that $A_{a}^{-}$is negative-invariant.

Now consider $x, y \in \Lambda$ arbitrary points. Since $A_{a}^{-}=\Lambda=A_{a}^{+}$, there exists $\Gamma_{a}^{+}(t, a) \in$ $A_{a}^{+}$a positive global trajectory, $\Gamma_{a}^{-}(t, a) \in A_{a}^{-}$a negative global trajectory and values $t_{x}>$ $0, t_{y}<0$ such that $\Gamma_{a}^{+}\left(t_{x}, a\right)=x$ and $\Gamma_{a}^{+}\left(t_{y}, a\right)=y$. Consequently there exists a global trajectory $\Gamma(t, y)$ passing through $y$ and $t^{*}=t_{x}+\left|t_{y}\right|>0$ such that $\Gamma\left(t^{*}, y\right)=x$.

LEMMA 4. Under the same hypotheses of Theorem 14, if any two points of $\Lambda$ can be connected by a global trajectory of $Z$, then $Z$ is chaotic on $\Lambda$.

Proof. The proof of Lemma 4 is similar to the proof of Theorem 8 by using Lemma 3 instead of Lemma 1.

Proof of Theorem 14. The proof is straightforward from Lemmas 3 and 4.

Remark 3. One should note that we cannot change the hypotheses of Theorem 14 by considering minimal sets instead of positive-minimal sets and negative-minimal sets simultaneously. Indeed, consider the PSVF $Z_{1}$ and the set $\Lambda_{1}$ as presented in Proposition 1. It holds that $\Lambda_{1}$ is minimal for $Z_{1}$. Nevertheless, $Z_{1}$ is not chaotic on $\Lambda_{1}$, since it is not topologically transitive on $\Lambda_{1}$. In order to see that, consider a non-empty open set $U$ located in $\Sigma^{+}$just above the sliding segment $S$ between $q$ and $p_{+}$in such way that all points of $U$ reach $S$ from $\Sigma^{+}$to $\Sigma$ and do not enter in the region $\Sigma^{-} \backslash \Sigma$. Consider also a non-empty open set $V$ under the same conditions of $U$, however located under $S$ on $\Sigma^{-}$. Thus, it is clear that all points of $U$ and $V$ reach $S$ and slides to $\partial \Lambda_{1}$ through the point $q$. However, since $\partial \Lambda_{1}$ is positive-minimal, it follows that the trajectories of $U$ and $V$ do not escape from $\partial \Lambda_{1}$ for positive values of time. Consequently we cannot connect points of $U$ and $V$ through a positive global trajectory and therefore $Z_{1}$ is not topologically transitive on $\Lambda_{1}$.

3.3. Conclusion. In this paper we have verified the existence of non-deterministic chaos in planar PSVFs without symmetry or presenting a canard structure. As far as the authors know, this is the first time that non-smooth systems with such characteristic are observed in the planar case. Moreover, we introduce definitions of minimal sets of PSVFs taking into account the fact that PSVFs have a strong dependence of the orientation of the trajectories, as we can see in Definition 1. Finally, we verify the presence of chaotic behavior in planar 
PSVFs and present a result relating chaotic behavior with orientable minimality, which emphasizes the importance of providing the definition of orientable minimality.

Acknowledgements. The first and second authors are partially supported by the CNPqBRAZIL grant 478230/2013-3. The second author is partially supported by the FAPESPBRAZIL grant 2012/00481-6 and 2014/02134-7. The third author is supported by the FAPESP-BRAZIL grant 2010/18015-6.

\section{REFERENCES}

[1] M. di Bernardo, C. J. Budd, A. R. Champneys and P. Kowalczyk. Piecewise-Smooth Dynamical Systems. Theory and Applications (Applied Mathematical Sciences, 163). Springer, London, 2008.

[2] C. A. Buzzi, T. de Carvalho and R. D. Euzebio. On Poincare-Bendixson theorem and non-trivial minimal sets in planar nonsmooth vector fields. Preprint, 2013, http://arxiv.org/pdf/1307.6825v1.pdf.

[3] A. Colombo and M. R. Jeffrey. Nondeterministic chaos, and the two-fold singularity in piecewise smooth flows. SIAM J. Appl. Dyn. Syst. 102 (2011), 423-451.

[4] R. L. Devaney. Introduction to Chaotic Dynamical Systems. Westview Press, Boulder, CO, 1989.

[5] A. F. Filippov. Differential equations with discontinuous righthand sides. Mathematics and its Applications (Soviet Series). Kluwer Academic Publishers, Dordrecht, 1988.

[6] M. Guardia, T. M. Seara and M. A. Teixeira. Generic bifurcations of low codimension of planar Filippov systems. J. Differential Equations 250 (2011), 1967-2023.

[7] C. Gutierrez. Smoothing continuous flows and the converse of Denjoy-Schwartz theorem. An. Acad. Brasil. Ci. 51(4) (1979), 581-589.

[8] M. R. Jeffrey. Nondeterminism in the limit of nonsmooth dynamics. Phys. Rev. Lett. 106 (2011),254103.

[9] m J. D. Meiss. Differential Dynamical Systems. SIAM, Philadelphia, PA, 2007.

[10] M. A. Teixeira. Perturbation Theory for Non-smooth Systems (Encyclopedia of Complexity and Systems Science). Ed. R. A. Meyers. Springer, New York, 2009, pp. 6697-6709. 